

\title{
A Semi-active Control-oriented Damper Model for an Automotive Suspension
}

\author{
Jorge de Jesus Lozoya-Santos, Olivier Sename, Luc Dugard, Rubén \\ Morales-Menéndez, Ricardo A. Ramirez-Mendoza
}

\section{To cite this version:}

Jorge de Jesus Lozoya-Santos, Olivier Sename, Luc Dugard, Rubén Morales-Menéndez, Ricardo A. Ramirez-Mendoza. A Semi-active Control-oriented Damper Model for an Automotive Suspension. AAC 2010 - 6th IFAC International Symposium on Advances in Automotive Control, Jul 2010, Munich, Germany. pp.P-16. hal-00504144

\section{HAL Id: hal-00504144 https://hal.science/hal-00504144}

Submitted on $20 \mathrm{Jul} 2010$

HAL is a multi-disciplinary open access archive for the deposit and dissemination of scientific research documents, whether they are published or not. The documents may come from teaching and research institutions in France or abroad, or from public or private research centers.
L'archive ouverte pluridisciplinaire HAL, est destinée au dépôt et à la diffusion de documents scientifiques de niveau recherche, publiés ou non, émanant des établissements d'enseignement et de recherche français ou étrangers, des laboratoires publics ou privés. 


\title{
A Semi-active Control-oriented Damper Model for an Automotive Suspension
}

\author{
Jorge de Jesus Lozoya-Santos ${ }^{* *}$ Olivier Sename* \\ Luc Dugard* Ruben Morales-Menendez ${ }^{* *}$ \\ Ricardo Ramirez-Mendoza** \\ * GIPSA-Lab, Control Systems Department, CNRS-Grenoble INP \\ ENSE3, BP 46, F-38402 St Martin d'Hères cedex, France \\ e-mail: \{olivier.sename, luc.dugard\}@gipsa-lab.grenoble-inp.fr \\ ** Tecnológico de Monterrey, 64849 Monterrey NL Mexico \\ e-mail: \{jorge.lozoya, rmm, ricardo.ramirez\}@itesm.mx
}

\begin{abstract}
This paper deals with the modelling of a magneto-rheological damper as a component of a quarter-car model. The objective is to provide an analysis of the transient and frequency behavior of the suspension system in order to evaluate the inherent linearities of two identified models: a complex one and a control-oriented one. The models have a common structure but the effect of the current and the dynamic behavior has different principle. The results show that the design of experiments is a key issue in the identification process. A quarter of a vehicle which includes a control-oriented damper model is identified as well-suited for the synthesis of controllers that perform well in terms of accurate frequency response when compared with that of a quarter-car with a semi-active suspension simulated with experimental data.
\end{abstract}

Keywords: Modelling, Identification, Semi-active Suspension, MR Dampers.

\section{INTRODUCTION}

The Magneto-Rheological $(M R)$ damper is a non-linear component with dissipative capability used in the control of semi-active suspensions, where the damping coefficient varies with to the applied electric current. The accurate modelling of the transient response and the force-velocity curve are not a trivial task. There are different approaches for modelling and simulation of this device as: physical meaning of the model parameters, Spencer Jr et al. (1997), parameters linked to the force-velocity curve, Guo et al. (2006), black-box approach, Savaresi et al. (2005). These models are able to simulate the non-linear, saturation and hysteresis behavior with good precision. The model approach considering the stiffness of the internal accumulator and static damping, Choi and Sung (2008), performs a good force magnitude estimation. However these models are not easy to use in the control design stage. Guo et al. (2006) propose an elegant and simple structure but the electric current is not present as an input. A common practice is to perform a polynomial dependence on the electric current of each parameter, obtaining good simulation results but this is very involved for control design.

This paper deals with:

(1) The comparison of two $M R$ damper models: a semiphenomenological and a control oriented model.

(2) The analysis of a quarter-car suspension model wellsuited for the synthesis of controllers that performs good tracking in terms of accurate frequency response

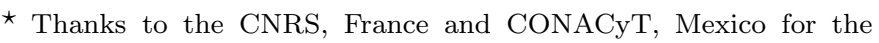
support under the Postgraduate Cooperation Project 2007-2011.
}

when compared with semi-active suspension simulated with experimental data.

The limitations (for controller synthesis) of the $M R$ damper models usually given in the literature are here overpassed through a $M R$ damper model which can be included in a quarter of vehicle $(Q o V)$ in a linear manner. The objective is then to provide an analysis of the transient and frequency behavior of the suspension system in order to evaluate the inherent linearities of two identified models: a complex one and a control-oriented one. The models have a common structure but the effect of the current on the $M R$ damper has a different principle. Four experimental dataset, two with the classical configuration in the literature and two with the new proposals are used to evaluate the models.

This paper is organized as follows. Section 2 specifies the semi-active damper, the experimental phase and the design of experiments. The semi-active modelling approaches are presented in section 3 . Section 4 discusses the influence of the experimental dataset on the identification. The frequency response of three quarter of vehicle using semiactive suspensions are analyzed on section 5 . Section 6 concludes the paper.

\section{THE SEMI-ACTIVE DAMPER AND THE EXPERIMENTAL SYSTEM}

The selected semi-active damper is a commercial $M R$ damper, which was tested in the automotive laboratory of Metalsa $^{1}$. This damper is a component of the intelligent suspension in the vehicles Cadillac ${ }^{T M}$ 2009, see Fig. 1.a.

\footnotetext{
1 www.metalsa.com.mx
} 
The experimental system is made up of three parts:

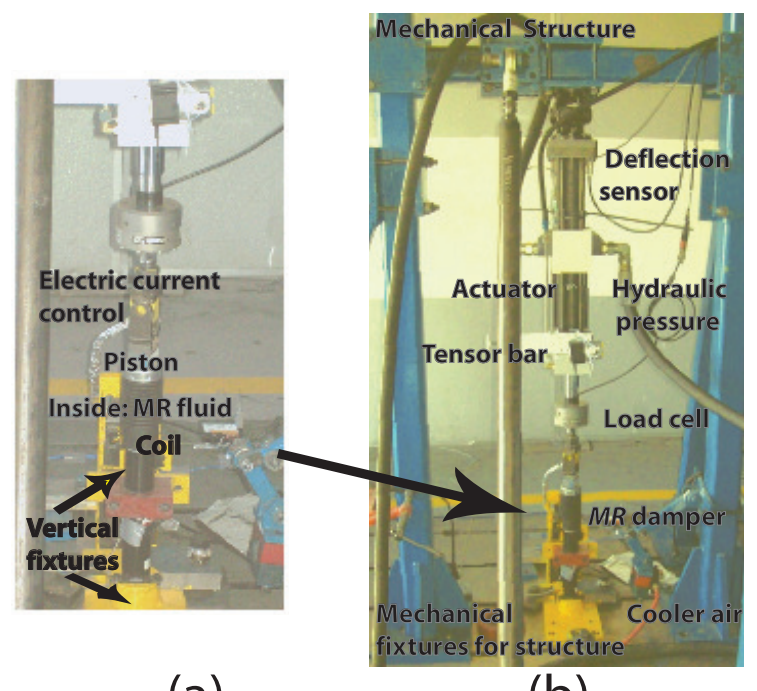

(a)

(b)

Fig. 1. $M R$ damper and experimental system details.

acquisition, actuation and control. The acquisition system captures 3 signals: (a) the displacement, (b) the generated force, and (c) the electric current through the $M R$ damper coil. The systems operation is done via a Supervisory Control System (SCS), which it is based on National Instruments ${ }^{T M}$ and LabView ${ }^{T M}$. The actuation system is a $M T S^{T M}$ equipment that includes: an actuator of 3000 PSI with a $15 \mathrm{~Hz}$ bandwidth, a controller hardware unit Flextest GT, a software Station Manager ${ }^{T M}$ and a MultiPurpose TestWare ${ }^{T M}$. The load capacity is $25 \mathrm{KN}$ at 3,000 psi. The stroke is $150 \mathrm{~mm}$, see Fig. 1.b. The span of the piston deflection is $\pm 12.5 \mathrm{~mm}$. An electric current driver adjusts the electric current. The span of the electric current command is from 0 to $2.5 \mathrm{~A}$, see Fig. 2 .

Four Design of Experiments $(D o E)$ for training are analyzed. The experiment inputs are: the displacement defined as the movement of the damper piston, and the electrical current through the coil. The output is the force delivered by the damper according to the inputs. The experiments are named experiment 1,2, 3 and 4 :

a. Experiment 1 is characterized by a displacement with amplitude modulation and increased clock period signal - (ICPS) electric current. 10 frequencies were tested in the span 1.40-14 Hz, see Fig. 2.

b. Experiment 2 is characterized by a Chirp sinusoidal displacement with constant current.

c. Experiment 3 is characterized by a Frequency modulated displacement and constant current.

d. Experiment 4 is characterized by a Frequency modulated displacement with ICPS electric current, getting two replicates.

For details on the DoEs, see Lozoya-Santos et al. (2009).

\section{SEMI-ACTIVE DAMPER MODELLING}

The $M R$ damper can be described by a dynamical model (eqn. 1) of a damper with a $M R$ fluid, equivalent to two dampers in parallel: a passive damper and a dynamic
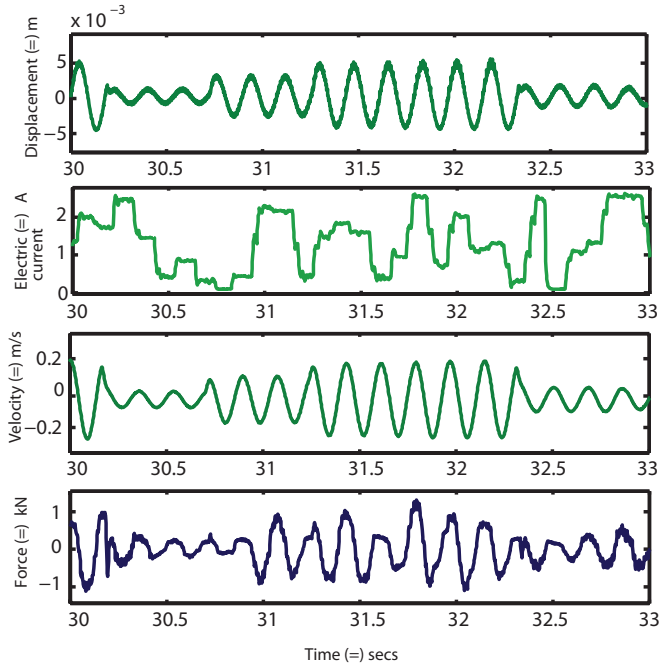

Fig. 2. Experimental dataset fragment from experiment 1. damper in the sense of a variable damping coefficient. The sum of the 2 components yields the $M R$ total damping force $f_{M R}$.

$$
\begin{aligned}
f_{M R} & =f_{p}(x, \dot{x})+f_{I}(x, \dot{x}, I) \\
f_{p} & =k_{e} x+c_{e} \dot{x} \\
f_{I} & =y_{f}(I) \cdot g(\dot{x}, x)
\end{aligned}
$$

where $x=z_{\text {def }}, \dot{x}=\dot{z}_{\text {def }}, f_{p}$ is the passive damping force, $I$ is the electric current through the coil, $f_{I}$ is the damping force due to $I, k_{e}$ is the passive stiffness coefficient, $c_{e}$ is the passive damping coefficient, $y_{f}$ is the yield point coefficient of $f_{I}, g(\cdot)$ is a nonlinear function.

This structure is used to study the action of the electric current on the $M R$ damper force.

\subsection{The Semi-Phenomenological (SP) model}

It captures the bi-viscous and hysteretic behavior in a smooth and concise form. The coefficients have a physical meaning, Guo et al. (2006):

$$
f_{M R}=a_{1} \tanh \left(a_{3}\left(\dot{x}+\frac{a_{4}}{a_{5}} x\right)\right)+a_{2}\left(\dot{x}+\frac{a_{4}}{a_{5}} x\right)
$$

where $a_{1}$ is the dynamic yield force $f_{M R}, a_{2}$ is the weight of the maximum piston velocity in order to compute the maximum generated force, i. e. post-yield viscous damping coefficient, $a_{3}$ is related to the pre-yield viscous damping coefficient respectively. $a_{4}$ and $a_{5}$ are the absolute values of the hysteretic critical velocity, and the hysteretic critical displacement respectively when $f_{M R}=0$. Each coefficient can be represented as a polynomial function of the electric current as:

$$
a_{i}(I)=\alpha_{5} I^{5}+\alpha_{4} I^{4}+\alpha_{3} I^{3}+\alpha_{2} I^{2}+\alpha_{1} I+\alpha_{0}
$$

where $\alpha_{i}$ are constant $\forall$ i.

\subsection{Control-oriented model: a modified SP version}

A modified version of the model (2) is presented where the force of the damper is described by: (a) the force due to the spring effect of the gas accumulator, (b) the damping 
force of the oil, and (c) the $M R$ force due to the electrical current. This is based on: first, the assumption of the null effect of the current on the pre-yield behavior, meaning that when the magnetic links in the $M R$ are broken by the stress, Jolly et al. (1999), the yield force stands quasi static until the links are joined, a phenomenon depending on the velocity. The second assumption is the proportional effect of the electric current on the force:

$$
f_{M R}=y_{M R} \cdot I \cdot \tanh \left(c_{M R} \dot{x}+k_{M R} x\right)+c_{p} \dot{x}+k_{p} x(4)
$$

where the constant parameters are: $y_{M R}$ is the yield force, $c_{M R}$ is the pre-yield damping coefficient, $k_{M R}$ is the postyield damping coefficient, $c_{p}$ and $k_{p}$ are the damping and stiffness coefficients for the passive damper.

\section{EXPERIMENTAL TESTS AND IDENTIFICATION}

\subsection{Exploration of the MR damper characteristic curve}

A comparison of the $D o E \mathrm{~s}$ in the $M R$ damper modelling is performed in order to define the framework scope of the obtained experimental dataset. Fig. 3.a shows the maximum deflection velocity achieved experimentally reported in the literature, Spencer Jr et al. (1997); Choi et al. (2001); Savaresi et al. (2005); Guo et al. (2006); Wang and Kamath (2006) and Shivaram and Gangadharan (2007). Most of the maximum velocities remain around $0.2 \mathrm{~m} / \mathrm{s}$ while the obtained experimental dataset used in this paper has a maximum velocity of $0.6 \mathrm{~m} / \mathrm{s}$, corresponding to the span of suspension deflection velocity for sedan vehicles, Bastow et al. (2004). The force-velocity curve for a typical damper shows 3 different linearity zones in rebound and 2 zones in compression as shown in Fig. 3.b. The higher velocity in the experimentation, the better the exploration of the $M R$ damping force on the various nonlinear zones. The literature experimental datasets remain in the first zone, while the experiments exposed in this paper explore two zones. They can be seen of Figure 5 as well, where the force velocity maps shows two damping coefficients for the preyield and postyield zones.

The experimental datasets shown in figures 4 and 5 are those for the lower and higher tested frequencies (1.4 and $14 \mathrm{~Hz}$ ) for the experiment 1 and the lower and higher electric currents $(0$ and $2.5 \mathrm{~Hz})$ for the experiment 2. These experiments show in a clear way the path of variation in the experimental characteristic curves when the $M R$ damper is submitted to input changes. The datasets obtained with the intermediate tested values of the frequencies and electric currents are not shown for clarity.

Summing up, the effects observed in the experimental dataset are:

(1) The applied current yields linear variation of the force.

(2) The variation of the displacement amplitude combined with the dynamic electric current ensures the exploration of the semi-active force-velocity plane while the larger the frequency of the sinusoidal displacement, the larger the maximum velocity achieved.

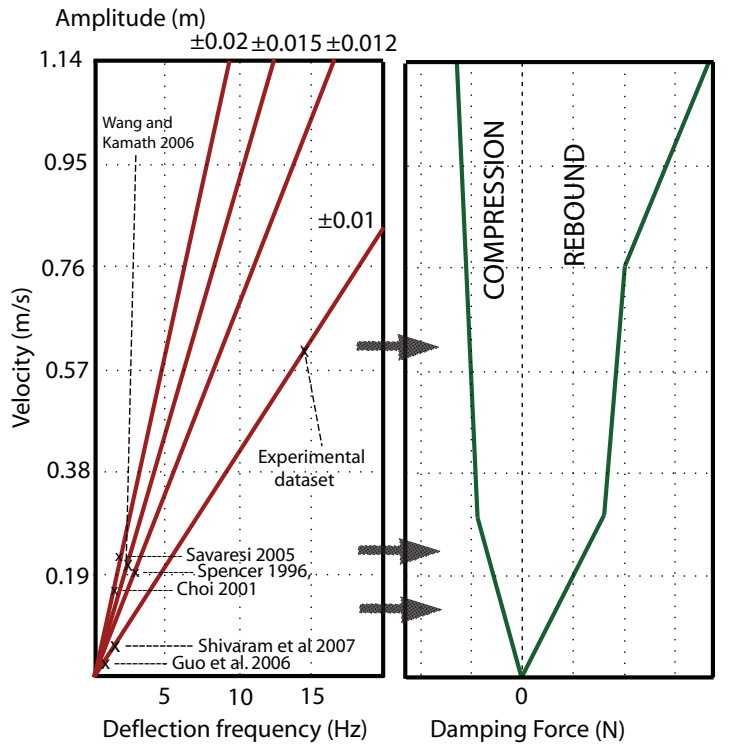

(a)

(b)

Fig. 3. Comparison of the $D o E$ results with the main contributions research $D o E$ s. The Fig. (a) shows the relation between the deflection frequency and amplitude with the deflection velocity. The Fig. (b) shows the relation force-deflection velocity. The higher the deflection velocity, the better the exploration of the nonlinear zones. The experimental datasets in this paper does a better exploration than the previous works.

(3) The classical approaches (i. e. experiments 2 and 3) with sinusoidal displacement and constant current get local information on the system.

4.2 Identification: parameter values depending on the experimental dataset

Each model is identified by using the nonlinear least square curve fit algorithm with the following cost function:

$$
\begin{array}{r}
\min _{\theta}\left\|\hat{f}_{M R}\left(\theta, \phi_{\text {dataset }}\right)-f_{M R}\right\|_{2}^{2}= \\
\min _{\theta} \sum_{i}\left(\hat{f}_{M R}\left(\theta, \phi_{i}\right)-f_{M R i}\right)
\end{array}
$$
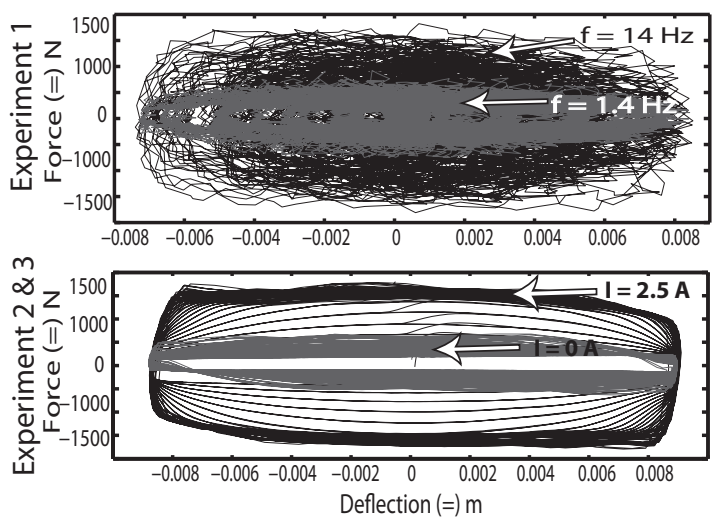

Fig. 4. Force-displacement curve of the tested $M R$ damper for the experiments 1 and 2 . 

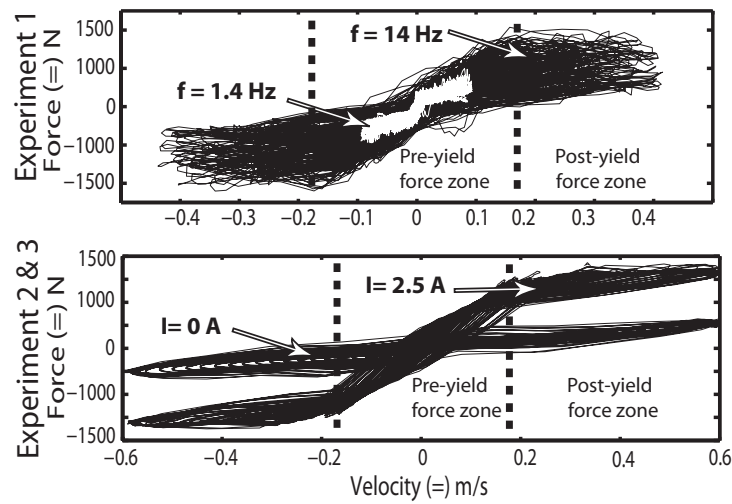

Fig. 5. Force-velocity curve of the tested $M R$ damper for the experiments 1 and 2 .

where $\theta$ is the vector of model parameters, $\phi_{\text {dataset }}$ the input dataset matrix, $\hat{f}_{M R}$ the predicted force, $f_{M R}$ the observed force, $i$ the $\mathrm{i}^{\text {th }}$ input dataset row, and $\phi_{\text {dataset }}=$ $[X, \dot{X}, I]$ where $X, \dot{X}$, and $I$ are the column vectors of the experimental dataset. After the identification of each model, the Error-to-Signal Ratio ESR and the parameters are recorded. Each model is identified with each experimental dataset. The obtained parameters values for each identification are compared in order to perform an analysis of the effect of the $D o E$ on their variations.

In order to observe the effects of the frequency content and of the electric current, the parameters of the semiphenomenological and control-oriented models are observed under two scenarios: in the first, the displacements consists in a rich frequency content with a constant sinusoidal amplitude and a constant electric current, (experiments 2 and 3), in the second, the sinusoidal displacements with random amplitude and fixed frequency are combined with the ICPS electric current (experiment 1).

The application of a constant electric current to a $M R$ damper allows the change of the dissipative features. Hence, for every electric current value, a different damper is obtained. Fig. 6 shows the change of the yield constant force $\left(a_{1}\right.$ or $\left.y_{M R}\right)$ and the pre-yield viscous damping coefficient $\left(a_{2}\right.$ or $\left.c_{p}\right)$ for both models. The trend is: the larger the electric current, the better the realistic meaning of the coefficient dampers. The plots show realistic values (in the sense of the physical meaning) from 0.8 to $2.5 \mathrm{~A}$. The experimental dataset for the experiment 3 at $0.8 \mathrm{~A}$ fits a control-oriented model with good generalized prediction but it shows some skewness when testing with other datasets. The rest of the experimental datasets identifies local models with this classical DoE approach because when they are tested with other datasets, the prediction is characterized by high deviations.

The information retrieved by a varying electric current paired with a displacement shaped by a sinusoidal wave with a constant frequency and random stepped amplitude is a key issue in the $M R$ damper identification. This feature avoids the frequency dependence of the model parameters. The results offer a realistic model parameterization, see Fig. 7. The models parameters show good convergence in the frequency range from 4.19 to $13.97 \mathrm{~Hz}$. Hence, the experiment 4 identifies generalized models of the $M R$ damper. Although the frequency displacement remains
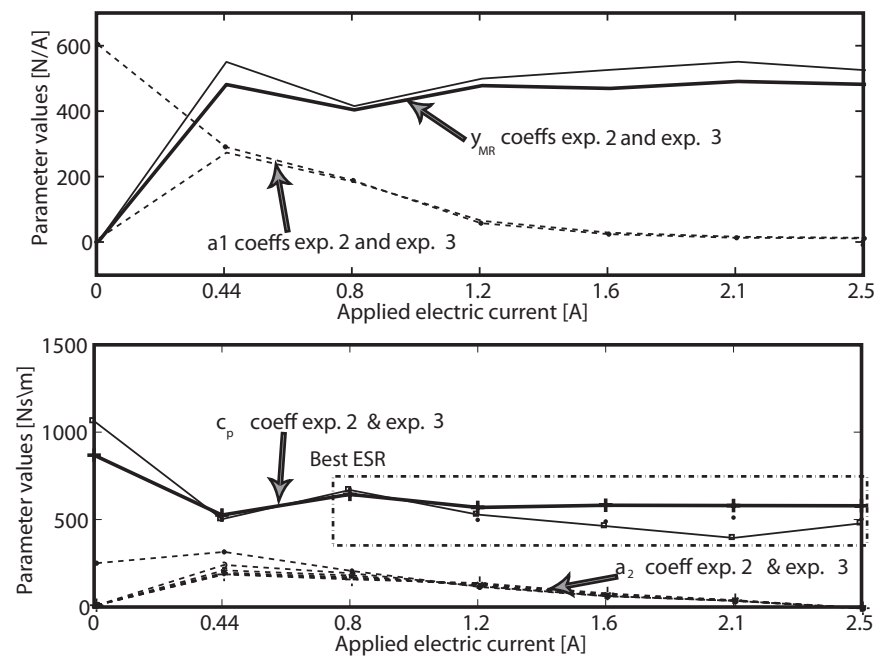

Fig. 6. Parameter variations when the models are fitted with experiments 2 and 3 . In the horizontal axes, each constant current corresponds to one dataset. $a_{1}$ and $a_{2}$ depends on the current (I). When $I \geq 0.8, y_{M R}$ and $c_{p}$ are constants.

constant, the fitted parameter values do not have great variations from one dataset to another.

The identified parameters have realistic physical meaning, the parameters of the best (lower ESR) control-oriented model are: $y_{M R} \approx 400 \mathrm{~N} / \mathrm{A}, c_{p} \approx 1117 \mathrm{~N} \cdot \mathrm{sec} / \mathrm{m}, c_{M R} \approx$ $9.2 \mathrm{sec} / \mathrm{m}, k_{p} \approx-2244 \mathrm{~N} / \mathrm{m}, k_{M R} \approx-18.51 / \mathrm{m}$ for the yield, pre-yield, and post-yield damping coefficients, and preyield and postyield stiffness coefficients. If the maximum velocity is $1.0 \mathrm{~m} / \mathrm{s}$ and the applied electric current $2.5 \mathrm{~A}$, the maximum force achievable with the control-oriented model is $f_{M R} \approx 2118 \mathrm{~N}$.

The values obtained for the parameter $c_{M R}$ in the controloriented model are shown in the Fig. 8. The value of $c_{M R}$ is $\approx 10 \pm 1$ which means that under post-yield actuation, the $M R$ damper force remains quasi-unchanged for variations in the velocity.

\section{PERFORMANCE ANALYSIS OF THE FREQUENCY RESPONSES OF SEMI-ACTIVE QUARTER OF VEHICLES}

The considered system consists of a simple model of a QoV with a semi-active damper used in Poussot-Vassal et al. (2008). The main components are the sprung mass $\left(m_{s}\right)$ and the unsprung mass $\left(m_{u s}\right)$. The spring with a stiffness coefficient $k_{s}$ and a semi-active damper built the suspension between masses. The stiffness coefficient $k_{t}$ models the wheel tire. The vertical position of the mass $m_{s}\left(m_{u s}\right)$ is defined by $z_{s}\left(z_{u s}\right)$. The variable $z_{r}$ is the road profile. It is assumed that the wheel contact is assured.

The dynamical equations of a quarter of vehicle are governed by:

$$
\left\{\begin{array}{l}
m_{s} \ddot{z}_{s}=-F_{\text {spring }}-F_{M R} \\
m_{u s} \ddot{z}_{u s}=F_{\text {spring }}+F_{M R}-k_{t}\left(z_{u s}-z_{r}\right)
\end{array}\right.
$$

where

$F_{\text {spring }}$ :spring force,

$F_{M R}: M R$ damper force, 



Fig. 7. Model parameter variations with experiments 1 and 4 for the polynomial and the control-oriented model. The plot shows the value of the parameters of each model. The legend Best ESR means that those parameter values performs the best prediction in each model. The dotted rectangle is the zone of generalized models, good generalizations but high ESR for low currents and low frequencies. The last two values in horizontal axes are the parameter values identified with experiment 4 .



Fig. 8. Variations of the control-oriented model parameter $c_{M R}$ fitted with the datasets of experiment 1 and 4. The last two values in horizontal axes are the parameter values identified with experiment 4

$z_{\text {def }}=z_{s}-z_{u s}$ : damper deflection (assumed to be measured or estimated),

$\dot{z}_{\text {def }}=\dot{z}_{s}-\dot{z}_{u s}$ :deflection velocity (directly computed from $\left.z_{\text {def }}\right)$.

The quarter of vehicle used in this paper is the Renault Megane Coupe ${ }^{T M}$ model (see Zin et al. (2006)) whose specific parameters are: $m_{s}=315 \mathrm{Kg}, m_{u s}=37.5 \mathrm{Kg}$, $k_{s}=29500 \mathrm{~N} / \mathrm{m}, k_{t}=210000 \mathrm{~N} / \mathrm{m}$. In the following three cases of quarter of vehicle are considered:

- Semi-Active QoV (SA-QoV): the $M R$ damper force is computed from the suspension deflection $z_{\text {def }}$ and the current $I$ using the static maps observed in the experimental data from 0 to $2.5 \mathrm{~A}$, Fig. 5 top plot.

- Semi-Phenomenological QoV (SP-QoV): the $M R$ damper force is computed according to $f_{M R}$ from the semi-phenomenological model (2) with polynomial dependence (3) on the current of each parameter, using a total of 28 parameters.
- Control-Oriented QoV (CO-QoV): the $M R$ damper force is computed according to $f_{M R}$ from the controloriented model (4) using a total of 5 parameters.

The frequency response is obtained with a pseudo-Bode procedure as in Poussot-Vassal et al. (2008). The chosen Transfer Functions $(T F)$ in the analysis are: $\ddot{z}_{s} / z_{r}$ used to evaluate the level of comfort, and $\left(z_{u s}-z_{r}\right) / z_{r}$ used to verify the road holding capability. The gain of the $T F$ $\ddot{z}_{s} / z_{r}$ must be small in the span of $2-10 \mathrm{~Hz}$. The gain of the $T F\left(z_{u s}-z_{r}\right) / z_{r}$ must always be close to 1 in order to guarantee a good vehicle handling.

The frequency responses of both CO-QoV and $S P-Q o V$ perform good prediction on the semi-active $Q o V$ behavior in the frequencies focused on comfort, Fig. 9. From the control point of view, the high frequency disturbances in the $M R$ force could be attenuated. When there is no current, the prediction of the acceleration in the sprung mass for frequencies below $2 \mathrm{~Hz}$ is quite accurate. A suggestion to challenge this, is the modeling, as uncertain parameters, of the $M R$ damper parameters $c_{p}$ and $k_{p}$. For $2.5 \mathrm{~A}, S P-Q o V$ presents high deviations in the prediction of the semi-active $Q o V$ while the $C O-Q o V$ performs a better following.
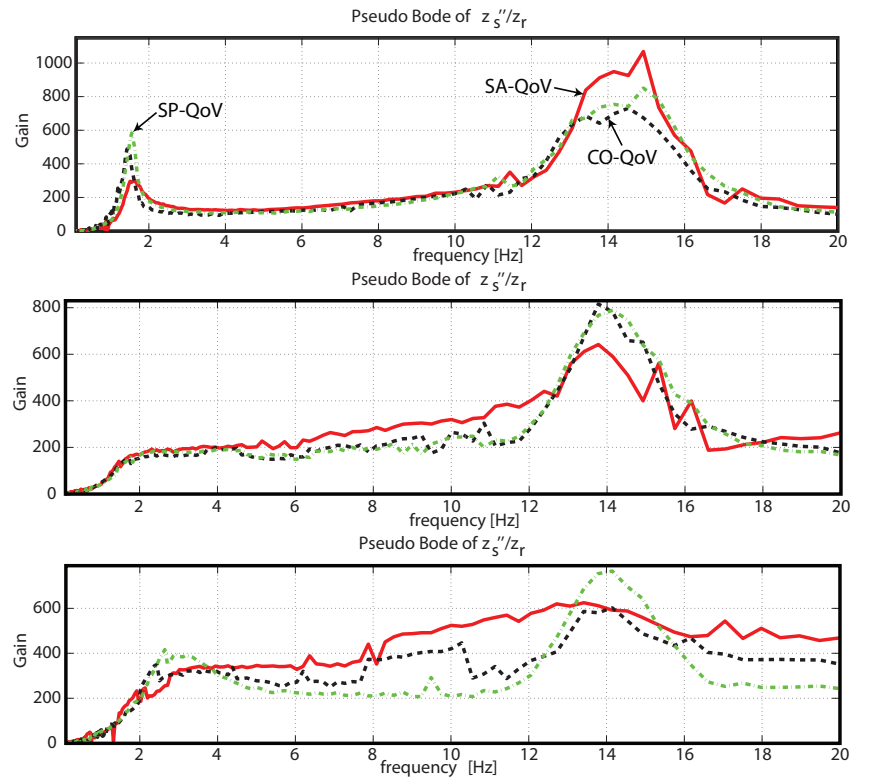

Fig. 9. Frequency response of $\ddot{z}_{s} / z_{r}$ for $0 \mathrm{~A}$ (top), $1 \mathrm{~A}$ (middle), and $2.5 \mathrm{~A}$ (bottom).

The simulation of the road holding measure has better accomplishment for both models, Fig. 10. When the current is $2.5 \mathrm{~A}$, the $S P-Q_{o} V$ shows high errors as for the transfer function $\ddot{z}_{s} / z_{r}$. Therefore, the polynomial dependence of the model parameters in semi-phenomenological model performs a good transient response, however, the result shows that the current-dependency of all the parameters could over estimate the damping force.

The control-oriented $Q_{o} V$ fits better to the semi-active QoV (see Table 1). Indeed, it approaches quite well the sprung mass acceleration $\ddot{z}_{s}$ and the deflection $z_{u s}-z_{r}$. The higher the current in the models, the better the area covering. 



Fig. 10. Frequency response of $\left(z_{u s}-z_{r}\right) / z_{r}$ for $0 \mathrm{~A}$ (top), $1 \mathrm{~A}$ (middle), and $2.5 \mathrm{~A}$ (bottom).

Table 1. Percentage error in pseudo-Bode of the quarter of vehicle for both models. The reference is the quarter of vehicle with the $M R$ damper experimental datasets.

\begin{tabular}{|c|c|c|c|}
\hline Transfer function & Current & $C O-Q o V$ & $S P-Q o V$ \\
\hline$\ddot{z}_{s} / z_{r}$ & $0 \mathrm{~A}$ & $-0.1148 \%$ & $-1.6491 \%$ \\
& $1 \mathrm{~A}$ & $1.898 \%$ & $2.2752 \%$ \\
& $2.5 \mathrm{~A}$ & $2.4531 \%$ & $2.6898 \%$ \\
\hline$\left(z_{u s}-z_{r}\right) / z_{r}$ & $0 \mathrm{~A}$ & $1.193 \%$ & $-0.31468 \%$ \\
& $1 \mathrm{~A}$ & $-14.644 \%$ & $-17.88 \%$ \\
& $2.5 \mathrm{~A}$ & $-8.4813 \%$ & $-13.242 \%$ \\
\hline
\end{tabular}

\section{CONCLUSION}

An analysis on the modelling, identification, and performances of a $M R$ damper in a semi-active suspension has been presented. The main results are: the $Q o V$ dynamics include a realistic behavior for the semi-active actuation, and the most important from the control point of view, the synthesis of closed-loop controllers for $Q o V$ could be possible. Also, it has been shown that the design of experiments is a key point in the identification of generalized models. The 5 parameter model of the $M R$ damper performs an excellent simulation behavior for the semiactive suspension in the frequencies of interest for comfort and road holding. The capability of variation with a linear input of the electric current, Fig. 11, in order to change the features of the automotive suspension allows the use of a linear-parameter varying $(L P V)$ model of a quarter of vehicle for control purposes.

\section{REFERENCES}

Bastow, D., Howard, G., and Whitehead, J.P. (2004). Car Suspension and Handling. SAE.

Choi, S.B., Lee, S.K., and Park, Y.P. (2001). A Hysteresis Model for Field-Dependent Damping Force of a Magnetorheological Damper. Sound and Vibration, 245, 375383.
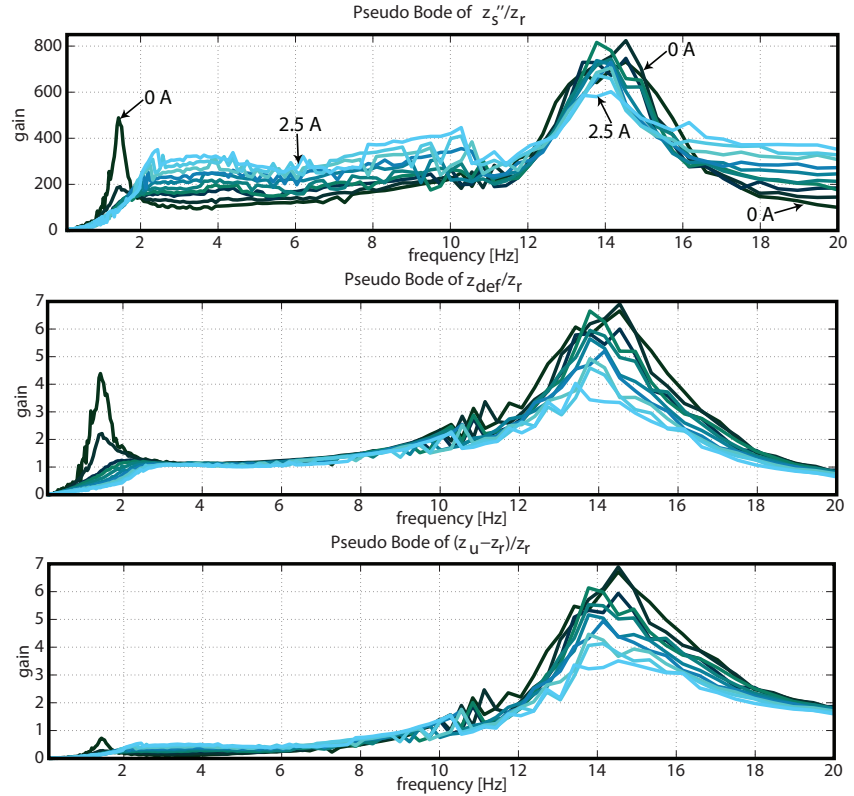

Fig. 11. Attitude of $M R$ damper control-oriented to modify the control objective (comfort or road holding) in a semi-active suspension in the span of $0-2.5$ electric current.

Choi, S.B. and Sung, K.G. (2008). Vibration Control of Magnetorheological Damper System subjected to Parameter Variations. Vehicle Design, 46(1), 94-110.

Guo, S., Yang, S., and Pan, C. (2006). Dynamical Modeling of Magneto-rheological Damper Behaviors. Int. Mater, Sys. and Struct., 17, 3-14.

Jolly, R., Bender, J.W., and Carlson, J.D. (1999). Properties and Applications of Commercial Magnetorheological Fluids. Technical report, Mark Thomas Lord Research Center.

Lozoya-Santos, J., Morales-Menendez, R., and RamirezMendoza, R. (2009). Design of Experiments for MR Damper Modelling. In Neural Netwotks, Int. Joint Conf. on, IEEE Proc.

Poussot-Vassal, C., Sename, O., Dugard, L., Gspr, P., Szab, Z., and Bokor, J. (2008). A New Semi-active Suspension Control Strategy through LPV Technique. Control Engineering Practice, 16(12), 1519-1534.

Savaresi, S.M., Bittanti, S., and Montiglio, M. (2005). Identification of Semi-Physical and Black-Box NonLinear Models: the Case of MR-Dampers for Vehicles Control. Automatica, 41(1), 113-127.

Shivaram, A.C. and Gangadharan, K.V. (2007). Statistical Modeling of a MR Fluid Damper using the Design of Experiments Approach. Smart Mater. and Struct., 16(4), 1310-1314.

Spencer Jr, B., Dyke, S., Sain, M., and Carlson, J. (1997). Phenomenological Model of a MR Damper. J. Engrg. Mech., 123(3), 230-238.

Wang, L.X. and Kamath, H. (2006). Modelling Hysteretic Behaviour in MR Fluids and Dampers using PhaseTransition Theory. Smart Mater. Struct., 15, 17251733.

Zin, A., Sename, O., Gaspar, P., Dugard, L., and Bokor, J. (2006). An LPV / $H_{\infty}$ Active Suspension Control for Global Chassis Technology: Design and Performance Analysis. In Proc of teh IEEE American Control Conf. 